\title{
Ambient noise correlations on a mobile, deformable array
}

\author{
Perry Naughton ${ }^{\text {a) }}$ \\ Department of Electrical and Computer Engineering, University of California, San Diego, \\ 9500 Gilman Drive, La Jolla, California 92093, USA \\ Philippe Roux \\ ISTerre, Université de Grenoble Alpes, CNRS, 38000 Grenoble, France \\ Riley Yeakle \\ Department of Electrical and Computer Engineering, University of California, San Diego, \\ 9500 Gilman Drive, La Jolla, California 92093, USA
}

Curt Schurgers

Qualcomm Institute, Calit2, University of California, San Diego, 9500 Gilman Drive, La Jolla, California 92093, USA

\author{
Ryan Kastner \\ Department of Computer Science and Engineering, University of California, San Diego, \\ 9500 Gilman Drive, La Jolla, California 92093, USA \\ Jules S. Jaffe and Paul L. D. Roberts \\ Scripps Institution of Oceanography, University of California San Diego, 9500 Gilman Drive, La Jolla, \\ California 92093-0238, USA
}

(Received 24 May 2016; revised 13 September 2016; accepted 16 November 2016; published online 15 December 2016)

\begin{abstract}
This paper presents a demonstration of ambient acoustic noise processing on a set of free floating oceanic receivers whose relative positions vary with time. It is shown that it is possible to retrieve information that is relevant to the travel time between the receivers. With thousands of short time cross-correlations (10 s) of varying distance, it is shown that on average, the decrease in amplitude of the noise correlation function with increased separation follows a power law. This suggests that there may be amplitude information that is embedded in the noise correlation function. An incoherent beamformer is developed, which shows that it is possible to determine a source direction using an array with moving elements and large element separation. This incoherent beamformer is used to verify cases when the distribution of noise sources in the ocean allows one to recover travel time information between pairs of mobile receivers. () 2016 Acoustical Society of America.
\end{abstract}

[http://dx.doi.org/10.1121/1.4971172]

[KGS]

Pages: $4260-4270$

\section{INTRODUCTION}

In the last decade, coherent processing of ambient acoustic noise developed into a new branch of ocean acoustic processing. ${ }^{1}$ Since the earliest demonstration of the emergence of coherent wavefronts from incoherent ambient acoustic noise ${ }^{2}$ there is better understanding of the theoretical mechanisms of this process ${ }^{3-6}$ and applications have been developed around this emergence. ${ }^{7-12}$ The emergence of coherent wavefronts is robust to the spatial and temporal variation of the acoustic environment in the ocean. However, current research activities are limited to arrays where the relative distance between elements is constant. ${ }^{1}$ Examples of these arrays are moored vertical ${ }^{2,13-15}$ or horizontal line arrays, ${ }^{7,9,13,16}$ or the mobile vertical array used as a passive fathometer. ${ }^{10-12}$ This paper demonstrates an application of a mobile array of subsea receivers that move independently of each other, resulting in an array with no preferred

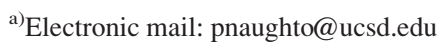

orientation. We discuss the challenges of this application, the limitations of an untethered array as well as the benefits.

The immediate result, and sometimes final goal, of coherent processing of ambient acoustic noise is the retrieval of the time domain Green's function (TDGF). The TDGF gives a description of the propagation paths between two receivers, in both directions. Typically, estimating the TDGF requires introducing a known active source into the environment. The important advance of ambient noise processing is that the estimation of the TDGF is done using ambient noise in the surrounding environment instead of introducing an active source. The spatial and temporal variation of the acoustic noise field in the ocean encouraged experiments spanning a diverse set of acoustic environments. Most of the literature on TDGF estimation relies on frequency bands that are dominated by shipping noise $(10 \mathrm{~Hz}<\mathrm{f}<1 \mathrm{kHz})^{2,13-15}$ These bands suffer the least from attenuation and contain the most power. This makes them ideal for estimating the TDGF over long distances. Noise from the surface of the ocean $(50<\mathrm{f}<5 \mathrm{kHz})$ was considered for a passive fathometer application. ${ }^{10-12}$ The benefit of surface noise for a 
fathometer is its vertical directionality, allowing the bottom reflection to be accurately retrieved. Biological sounds were considered (from a species of croaker fish), ${ }^{7,16}$ using higher frequencies $(350-700 \mathrm{~Hz})$ and closer distances $(1-50 \mathrm{~m})$. Low frequencies $(<100 \mathrm{~Hz})$ were considered in the deep ocean. ${ }^{8,9,17}$ In general, tens of minutes of noise has been used to recover the arrival structure of the TDGF over distances from hundreds of meters to a few kilometers.

Recovering the TDGF is the first step of many applications. Using estimates of the direct acoustic path recovered with the TDGF, both the clock bias and relative geometry of an array can be formulated as a nonlinear optimization problem and estimated, ${ }^{5}$ a process termed array element self-synchronization and array element self-localization, respectively. Synchronizing receivers using the TDGF was also demonstrated for seismic sensors. ${ }^{18}$ Acoustic thermometry was proposed to measure the changing temperatures in the deep ocean by estimating changes in the speed of sound (which is largely influenced by temperature) through the arrival structure of the TDGF. ${ }^{8,9}$ A passive fathometer was implemented to measure the depth of the ocean and seabed layering using ambient noise by extracting the time value of the bottom reflected path for vertically propagating ambient noise from noise excitation at the surface. ${ }^{10-12}$ In the fathometer implementation, delay sum beamforming was used to leverage multiple elements in a vertical array to enhance the emergence of the TDGF in the vertical direction. ${ }^{12}$ More theoretical developments were made $^{10}$ and an adaptive beamforming procedure was proposed to enhance the result of the original formulation. ${ }^{11}$ Similar to the vertical beamforming of the passive fathometer, beamforming to enhance the emergence of horizontal coherent arrivals was proposed by Leroy et al. ${ }^{15}$ and shown to enhance the signal to noise ratio of the noise correlation function using frequency bands dominated by horizontally traveling shipping noise. ${ }^{14}$ This beamforming procedure enabled short range tomography by reinforcing the most stable horizontal ray between the receivers. ${ }^{14}$

There are two competing forces in the emergence of the TDGF. There is an inherent variance in the noise correlation function $(\mathrm{NCF})$ resulting from the random distribution of noise sources contributing to the NCF. This variance is inversely proportional to the time-bandwidth product under the assumption of an isotropic noise distribution. ${ }^{4}$ Given a stationary environment, a longer time window results in a higher signal to noise ratio by decreasing the variance of the NCF. Similarly, longer time windows result in more coherent contributions that build the TDGF. Unfortunately, the acoustic environment in the ocean is not stationary. Changes in the propagation of sound between two receivers degrades the emergence of the TDGF. ${ }^{16}$ These temporal changes in the acoustic environment are the limiting factor in the successful recovery of the TDGF.

In this work we consider ambient noise processing on subsea receivers that float freely with the currents. The motion of the receivers is the main change in propagation paths between receivers. This motion and resulting deformation of the array severely restricts the length of the correlation window we can use and makes recovering the TDGF difficult. Despite these challenges, we demonstrate that we can recover information that is relevant to the propagation time between the two receivers. In some cases, this propagation information matches with the arrival structure of the TDGF. Using thousands of measurements from short time cross-correlations, we provide an analysis of how the amplitude of the recovered TDGF compares to the theoretical amplitude. Additionally, we show that we can use this deformable array to detect dominant source directions in the ambient noise field and describe how these detected directions are consistent with our analysis of being able to recover the arrival information of the TDGF. These results suggest the possibility of source localization using a mobile deformable array.

\section{METHODS}

\section{A. Autonomous underwater explorers}

The experiment leverages autonomous underwater explorers (AUEs), designed and built at the Scripps Institute of Oceanography to increase underwater sampling resolution in both space and time. ${ }^{25}$ Each AUE is a buoyancy controlled unit that can track a depth profile in the ocean's water column by adjusting its buoyancy. The AUEs have no actuation in the horizontal direction, are fully at the mercy of the currents and can move substantially while deployed in the ocean. AUEs collect acoustic data from an HTI-96-MIN hydrophone, accelerometer data, temperature, and pressure data.

The depth of the AUE is determined using the on board pressure sensor. To localize each AUE in the other two directions (latitude and longitude), an acoustic triangulation system is set up to act similar to a GPS system. Five buoys are positioned on the surface of the ocean to send a linear modulated chirp in the frequency range $8-15 \mathrm{kHz}$ to be received by the hydrophone of each AUE. Each of five buoys takes turns pinging and each packet of five pings occurs around every ten seconds. The AUEs have limited on-board processing and no communication infrastructure, so all localization is done offline after they are retrieved. Since the AUEs can move in the period that the acoustic signals are being received, we can either assume that they are stationary during the buoy signal acquisition or we can estimate their motion during the signal reception by employing a factor graph framework. ${ }^{19,20}$ We have performed both methods for this dataset and they yield similar trajectories. These estimated trajectories are used throughout the analysis given in this paper. To maintain their depth, each AUE uses a motor to drive a piston that changes its buoyancy. When the motor is on, the hydrophones are saturated and we have no usable acoustic signal for that time. We observe the motors to be on for at least $10 \%$ of the duration of the experiment. The clocks of the AUE are synchronized at the beginning and end of the experiment using a GPS receiver on each AUE, and a linear clock drift model is applied to each AUE clock before any processing on the acoustic data is performed. 


\section{B. Experiment}

The experiment makes use of data that was collected off of the coast of San Diego in October of 2013. The purpose of this experiment was to validate the trajectory estimation of the AUEs as well as measure currents and internal waves in the ocean. For the experiment, 13 AUEs tracked a depth of $10 \mathrm{~m}$ and drifted with the currents for $5 \mathrm{~h}$. A wirewalker ${ }^{21}$ was deployed near the array which collected temperature and pressure data. From the wirewalker data, it was determined that the AUEs were sitting on a steep thermocline and the speed of sound was determined to be $1519 \mathrm{~m} / \mathrm{s}$ at $10 \mathrm{~m}$ depth (more discussion of the acoustic environment during the deployment is found in Fig. 6 and in the propagation environment section). Figure 1 shows the trajectories of the AUEs and the position of each of the five buoys in relation to the trajectories. The trajectories of AUE 7, 8, and 13 are highlighted; we will be using these trajectories in our analysis. It is important to note that these trajectories have no notion of time meaning that it is difficult to differentiate when the AUEs crossed paths in the experiment from the times where the AUE crossed paths with an old position of another AUE.

\section{NOISE CORRELATION FUNCTION}

\section{A. Theory}

We are interested in the feasibility of extracting the TDGF between a pair of mobile receivers through a crosscorrelation of ambient ocean acoustic noise. Practically, the normalized NCF between signals $p_{i}(t)$ and $p_{j}(t)$ collected at receiver $i$ and $j$, respectively, is computed as

$$
\begin{aligned}
C_{i, j}(\tau)= & \int_{t_{c}-T_{r} / 2}^{t_{c}+T_{r} / 2} p_{i}(t) p_{j}(t+\tau) d t / \sqrt{\int_{t_{c}-T_{r} / 2}^{t_{c}+T_{r} / 2} p_{i}(t)^{2} d t} \\
& \times \sqrt{\int_{t_{c}-T_{r} / 2}^{t_{c}+T_{r} / 2} p_{j}(t)^{2} d t},
\end{aligned}
$$

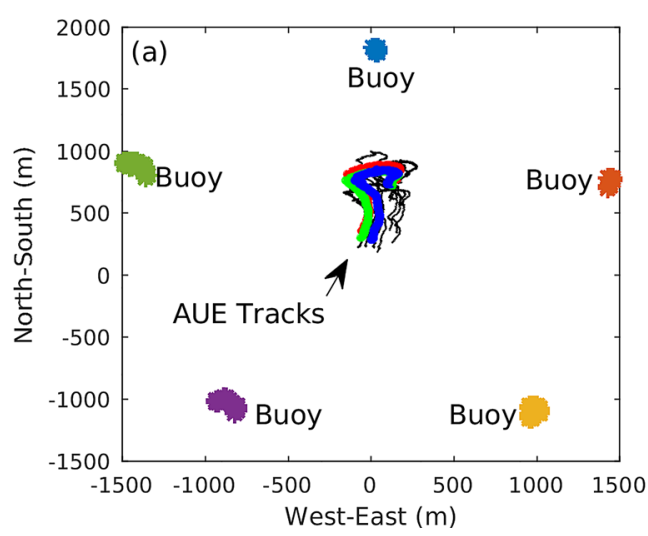

where $t_{c}$ controls the center time of the signal and $T_{r}$ the length of the correlation window. With respect to ocean acoustics, it has been shown both experimentally ${ }^{2}$ and theoretically ${ }^{3}$ that the NCF yields an estimate of the arrival structure of the TDGF (the amplitude of the TDGF is generally not recovered). The intuition behind this estimate is that the wavefield components of the ambient noise field that travel through both receivers average coherently while the components of the noise field that only travel through one receiver average incoherently. Sufficient time averaging, either by controlling the time window parameter $\left(T_{r}\right)$ or by averaging several time-gated windows, is needed to ensure that there are components of the noise field that pass through both receivers. Otherwise the peaks of the TDGF will not emerge. In previous works, the length of the time window is generally on the order of $10-30 \mathrm{~min}$ for ocean noise. $^{2,4,7,13,15}$ The length of the time window is largely determined by the noise distribution in the array's environment and the frequency band chosen, which determines how isotropic the noise field is. For a specific acoustic environment with a horizontal array (element spacing between 1 and $120 \mathrm{~m}$ ) in shallow water, the optimal time average was determined to be $3 \mathrm{~h}$ and $50 \mathrm{~min}$ before changes in the acoustic environment started to degrade the estimate of the TDGF. $^{16}$

The emergence rate of the TDGF has been studied in past work and it was determined that the variance of the normalized NCF is $\left(2 B_{\omega} T_{r}\right)^{-1}$ for an isotropic distribution of noise sources. ${ }^{7}$ This is the noise floor that we are trying to overcome to see an emergence of the direct path in the TDGF. The amplitude of the direct path of the TDGF between two receivers depends on their separation as well as the acoustic environment. Simplified models of the acoustic environment indicate that the amplitude of the TDGF is related to the receiver separation, $R$, either by $1 / R$ (through cylindrical spreading) or $1 / R^{2}$ (through spherical spreading). This gives us a rough rule of thumb for the relationship between the length of time, $T_{r}$, needed to see an emergence

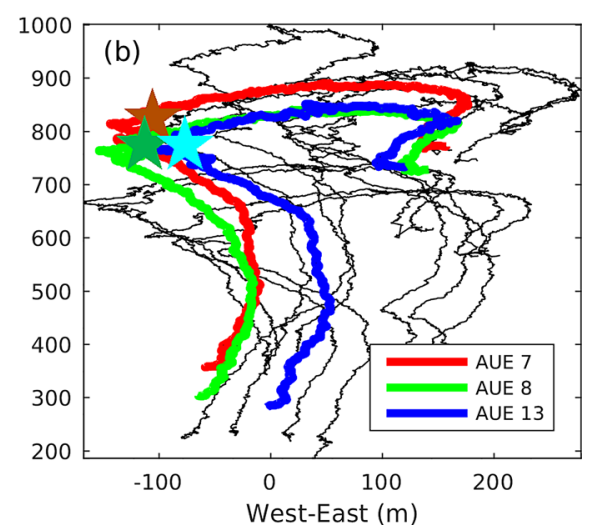

FIG. 1. (Color online) Experiment setup: (a) 5 buoys are positioned on the surface of the ocean along the perimeter of a deployment. These buoys collect GPS data and take turns transmitting a linearly modulated chirp. The AUEs, positioned in the center of the buoy array, measure the time of arrival of these chirps and we compute estimates of their positions based on these signals. (b) A close up of the trajectories of the AUEs with three individual AUEs highlighted. We notice that the trajectories are rough because they are computed at discrete times with some noise. It is also difficult to deduce the time element of these trajectories. Where it looks like AUEs cross paths it is usually the case that an AUE is crossing the path of an older position of another AUE. To demonstrate this we plot the positions of the three highlighted AUEs with a star during one time in the deployment. From this we can see that AUE 7 is north of AUE 8 at this time even though it may look like AUE 7 and 8 are crossing paths if we were to only look at their trajectories. 
based on the receiver separation, $R$. Given twice the separation between receivers one must double or quadruple the time window in order to keep the signal to noise ratio constant, depending on the environment. Unfortunately, short time windows also make the NCF vulnerable to nearby dominant sources because there is not enough time to average these sources out (see Fig. 3 in Roux et $\mathrm{al}^{2}{ }^{2}$ ). Dominant sources can bias both the amplitude and the arrival time of the peak in the NCF.

The key challenge presented in this work is that the receivers are moving while they are deployed and the relative distances between the receivers are constantly changing. This prevents us from using long time averages of the crosscorrelations that were used in previous literature to estimate the TDGF. This challenge has been mentioned before, where estimates of the TDGF were less reliable on top of a vertical array than on the bottom of the array because the anchor at the bottom of the array allowed less movement than at the top. ${ }^{15}$ In our scenario, this problem is magnified by the fact that the receivers were designed to be Lagrangian and float freely with the currents. In fact, the AUEs were designed to study the small scale current fluctuations or the relative motion between the AUEs as opposed to the group motion (i.e., the average motion of the AUEs). In our formulation, we assume that the receivers are stationary for small segments of time so that we can use the theory developed in previous work. Even though there are local changes in the distances between the receivers, the movement between receivers is strongly correlated (driven by the currents). This helps keep the array together and we can assume that the receivers will be in the vicinity of each other during the entire deployment.

\section{B. NCF results}

To compute the NCF, several preprocessing steps are executed before the correlation takes place. The first preprocessing step is to "whiten" the signal's frequency spectrum, meaning equal power is assigned to each frequency band. ${ }^{4,13,15,16}$ This helps spread the contribution to the NCF across each frequency band instead of having the NCF be dominated by the frequency bands with the most power. Next, time series values with high amplitudes are truncated to the fourth standard deviation of the $p_{i}(t)$ and $p_{j}(t)$ signals. Last, each signal is normalized by the energy of the signal so that the peak of the auto-correlation of each signal would have amplitude 1 . The purpose of these preprocessing steps is to ensure that peaks in the NCF come from coherent contributions of many sources instead of contributions from a few high energy sources which would likely bias the TDGF estimate, and to normalize each time series so that comparisons at different times can be made. More insight into these preprocessing steps can be found in Refs. 4, 13, 15, and 16.

Figures 2(a) and 2(b) show the NCF of receiver 8 and 13 and receiver 7 and 8 , respectively, for the entire deployment time. These two pairs were chosen as examples to represent all possible permutations of AUE pairs in the array. For our experiments, we take the value of $T_{r}=10 \mathrm{~s}$ (more discussion on the choice of $T_{r}$ and $B_{\omega}$ can be found in Sec. IIIC) in Eq. (1) and $t_{c}$ is taken to
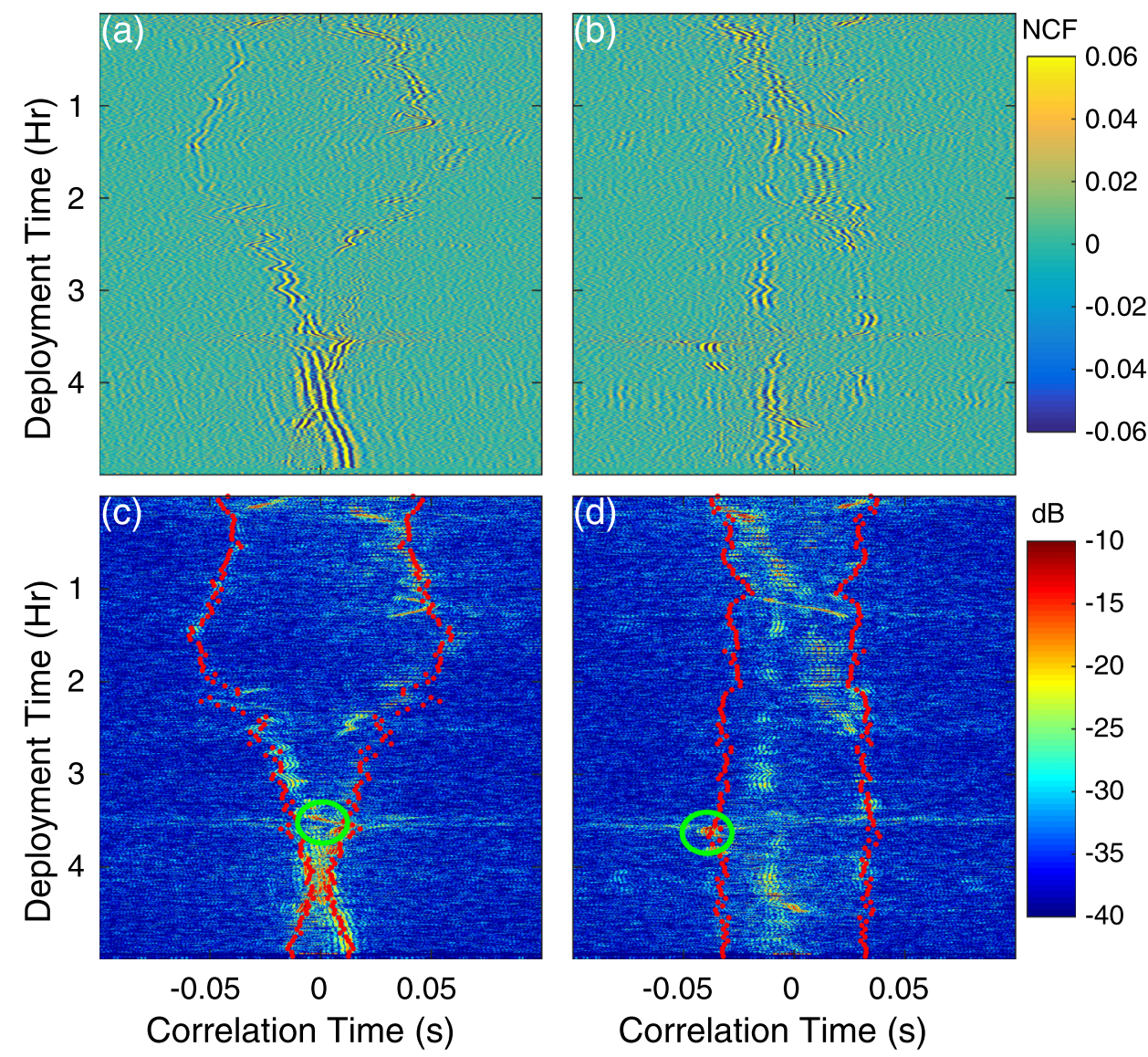

FIG. 2. (Color online) Noise correlations: Left-AUE 8 and 13, right-AUE 7 and 8. (a)-(b) Short time crosscorrelations of ambient acoustic noise between AUE pairs ( $x$ axis) at different times during the AUE deployment $(y$ axis). The color represents the amplitude of the NCF, defined in Eq. (1). (c)-(d) The intensity (in $\mathrm{dB}$ ) of the NCF with the estimated distances of the AUEs (generated from Fig. 1) plotted over the intensity. From (a)-(d) we can see that for some pairs the peaks of the NCF align with the direct path between the pair of AUEs, for others the peaks do not show agreement with the distance between the pairs. We suggest that there is a directionality in the ambient noise field, and those pairs with their end-fire beams aligned with the dominant direction show the symmetry we would expect from the TDGF while those pairs whose endfire beam do not align do not show the symmetry. The green circles demonstrate a time when there is a change in the dominant noise direction of the ambient noise field, and we can see that the peaks match the distance for AUEs 7 and 8 this time and not AUEs 8 and 13. (This is described more in Fig. 9.) Parameters for correlations: time window length- $10 \mathrm{~s}$, bandwidth-[100-500 Hz]. 
be integer multiples of $15 \mathrm{~s}$. There is no overlap of data used between subsequent computations of the NCF. In each of these figures, the y-axis represents the value of $t_{c}$, a measure of the deployment time (in hours), while the $x$ axis denotes the cross-correlation time (in seconds). For each correlation we used the frequency band $B_{\omega}$ $=[100,500] \mathrm{Hz}$ whitened according to the preprocessing procedure described above.

If we were to see the emergence of the TDGF we would expect a symmetry in the NCF around zero correlation time (it will be centered around zero only if the receivers are synchronized $^{7}$ ). The peaks of the NCF would represent the causal and anti-causal solutions to the TDGF, meaning the peaks of the NCF would demonstrate the acoustic paths between the two receivers, including the direct and possibly reflected paths. In positive correlation time we would see the paths traveling from one receiver to the other, and in negative correlation time the paths would be traveling the opposite direction.

Figures 2(c) and 2(d) show the intensity of the NCF with the estimated travel time (from the computed positions of each receiver) plotted over the intensity to demonstrate the similarity of the NCF with the direct path between the two receivers. These distances were derived using the information shown in Fig. 1. If the NCF was a representation of the TDGF, the correlation peaks would be related to the direct path shown. We see a difference in behavior between the two different pairs of receivers. The first pair, receivers 8 and 13, shows the symmetric correlation function that we would expect from the emergence of the TDGF. The symmetry follows the change in the direct path between the two receivers; as the receivers move closer together the peaks in the NCF move closer together. Not only are the trends similar but the time value of the peak frequently matches the travel time between the receivers.

In the frame of previous work, the results presented in Figs. 2(a) and 2(c) are unique. We see a symmetric structure of the NCF that frequently matches the direct acoustic path. This is using a short correlation window, only ten seconds, compared to the tens of minutes or hours of previous work. This result shows promise that the first arrival time of the TDGF can be recovered using short time windows on receivers that are moving relative to each other.

The second pair (receivers 7 and 8) does not show the symmetric structure that we would expect from the TDGF and the peaks in the NCF do not match the direct path that was derived from the distance between the receivers. From a simple analysis of which pairs show the symmetric structure and which do not (for the pairs shown as well as pairs not shown) we attribute the difference in results to directionality in the noise field. Referring back to Fig. 1, the relative geometry of the pairs of receivers shown by the stars are relatively consistent during the deployment. Pairs whose endfire beam (the endfire beam is defined by the ray that passes through both receivers) is mostly east-west demonstrates the symmetry described for the receiver pair 8 and 13, while receivers whose endfire beam aligns with north-south do not demonstrate the symmetry described. We can also see some directionality in the ambient noise field by examining pair 8 and
13. In this correlation, there is an imbalance in the magnitude of the peaks in the NCF. The peaks in positive correlation time are larger than the peaks in negative correlation time. From this, we can determine that most of the acoustic energy is being received from the east. The peaks in negative correlation time indicate that there are acoustic wavefronts that are propagating from the west, but the smaller magnitude of these peaks indicates less acoustic energy from this direction.

We further quantify this observation by computing the residual describing the difference between the arrival time of the maximum of the NCF and the expected arrival time. We compute these residuals for all pairs. Figure 3(a) shows an example for one pair. We are comparing the time value of the maximum (shown with blue dots) with the expected path (shown with the red dots). The maximum of the NCF is computed for both positive and negative correlation times for each time step and the orientation of the AUE pair is used to match the positive and negative correlation times with a direction of incidence noise. Figures 3(b)-3(d) describe cumulative distribution of residuals for different directions of ambient noise. These plots are shown for different times in the deployment.

The results shown in Figs. 3(b)-3(d) demonstrate two important points about retrieving the arrival structure of the TDGF from ambient noise during this experiment. First, the noise field is anisotropic. Different source directions produce different distributions of residuals. For example, Fig. 3(b) shows that the peaks that result from noise coming from the east match the expected TDGF the best (a greater percentage of peaks have smaller residuals) during the time period hour 0.5 -hour 1.5 . The second point is that the ambient noise field is time varying. The residuals do not follow similar trends when viewed across different times during the deployment. The smaller residuals for the Easterly ambient noise of Fig. 3(b) are not found in Fig. 3(c) (hour 2-hour 2.5). Also, Fig. 3(d) shows a time in the deployment where ambient noise from the north provides accurate arrival times when compared to the other two times. Over all the deployment times, we can see that the smallest residuals occur during the time period hour 0.5 -hour 1.5 with noise coming from the esterly direction. The peaks resulting from easterly noise at these times match the expected arrival structure with millisecond accuracy $10 \%$ of the time and match with $10 \mathrm{~ms}$ accuracy around $40 \%$ of the time. In evaluating these numbers, it is important to understand that the positions of the AUEs are also estimates that are prone to some error. We do not expect to be able to estimate the direct arrival between the AUEs with less than millisecond accuracy.

The discrepancies in the arrival time during different deployment times points to a difficulty in ambient noise processing. We can see that in Fig. 2(a) there are times where the NCF is single sided [see Fig. 2(a) around $1 \mathrm{~h}$ ] and even times when there seem to be no peaks at all [see Fig. 2(a) slightly after $2 \mathrm{~h}$ ]. This could either be a result of anisotropic noise fields; specifically nonstationary shipping events or it could be the result of interferers passing through the endfire region of a receiver pair. For lower frequencies that are typically dominated by shipping noise, coherent arrivals typically occur 

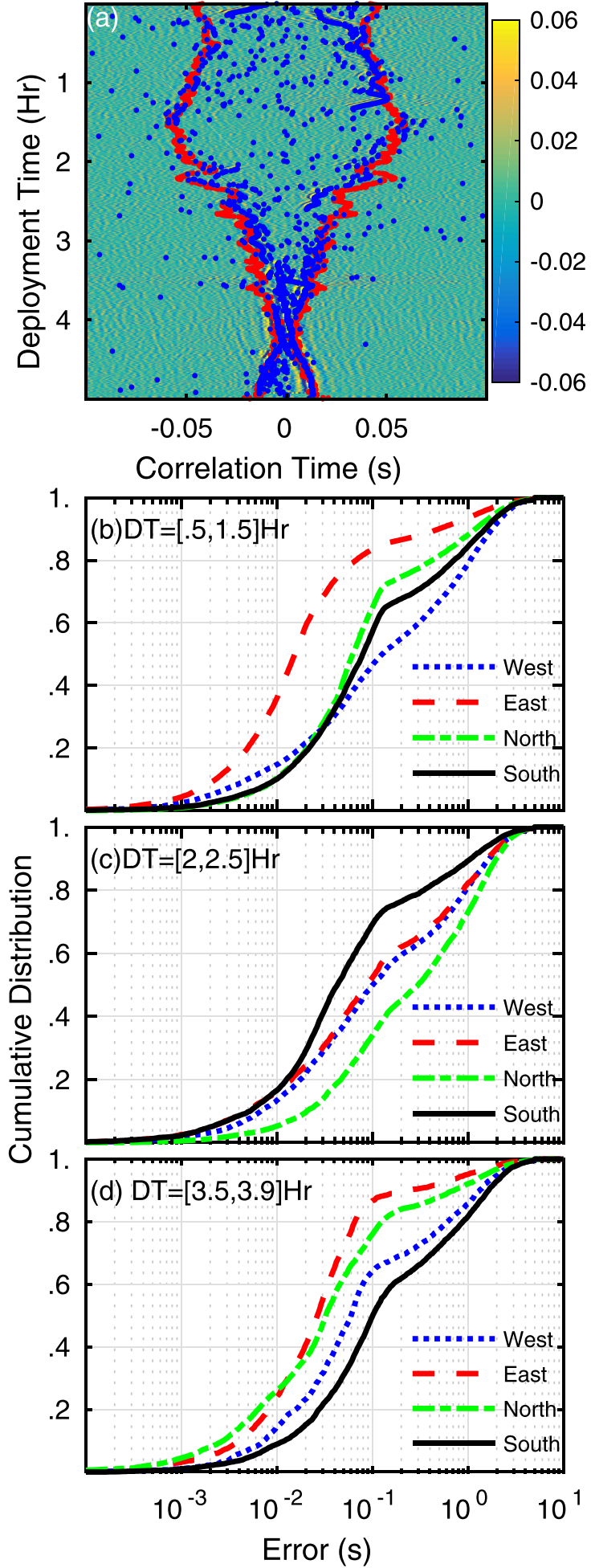

FIG. 3. (Color online) Arrival time accuracy analysis. Quantitative evaluation of the accuracy of the arrival time of the NCF peaks compared to the expected direct acoustic path (a) The maximum values of the NCF are shown for each center time, $t_{c}$, in both positive and negative correlation time (blue dots). The expected peaks of the NCF are also shown (red dots). (b)-(d) Cumulative distribution as a function of the difference in predicted direct path and the maximum value of each correlation peak (computed for all pairs of floats). The correlation peaks are separated so that a peak corresponds to an approximate direction of ambient noise sources that would generate that peak. These plots are shown for different deployment times (DTs). (b) DTs between 0.5 and $1.5 \mathrm{~h}$. (c) DTs between 2 and $2.5 \mathrm{~h}$. (d) DTs between 3.5 and $3.9 \mathrm{~h}$. These plots give a feel for both the accuracy of the arrival times as a function of distance as well as the time evolution of the ambient noise field during the $5 \mathrm{~h}$ experiment. during discrete times [see Fig. 2(e) in Ref. 14]. The long time windows of past works are chosen to make the noise fields act more stationary and more isotropic. There is a greater chance of coherent arrivals passing through both receivers when the NCF includes longer time periods. An important result of ambient noise processing is that arrival information of the TDGF can still be recovered even when the noise field is far from the isotropic assumption. In this work, we are even further from the isotropic assumption because the receiver movement restricts the time window. We can see the effect of this in the results shown. Some pairs of floats are completely biased, such as the pair shown in Figs. 2(b) and 2(d). Even the pairs that do show the symmetric structure of the TDGF have times at which they are biased, such as the pair shown in Figs. 2(a) and 2(c).

Techniques have been investigated to overcome the difficulties of anisotropic noise that result in the incorrect arrival times of the NCF shown in Fig. 2(d). Specifically, in seismic studies, ${ }^{22,23}$ a second-order correlation process is defined that uses surrounding receivers to improve the TDGF estimate of a given receiver pair. This second order correlation process benefits from scattering on seismic heterogeneities, and uses the other receivers in the array to act as secondary sources. This processes relies on significant reverberation and scattering in order to accumulate noise directions that are different from the dominant source directions. Unfortunately, the acoustic environment of the ocean does not permit enough scattering for this second-order correlation process to overcome anisotropy. We performed this second order correlation process on the data shown in Fig. 2 and the results were similar to NCF.

\section{Choosing $T_{r}$ and $B_{\omega}$}

In Sec. IIIB, we presented results for computing the NCF for only one choice of $T_{r}$ and $B_{\omega}$. The choice of $T_{r}$ and $B_{\omega}$ along with the relative velocity of the receivers determines the validity of our short time stationary assumption. In order for a wavefront with wavelength $\lambda$ to contribute coherently to a peak in the NCF, the relative distance between receivers must change less than $\lambda / 2$. This condition constrains both $B_{\omega}$, through the wavelengths being correlated, and $T_{r}$ through the amount of relative movement that can occur between the receivers (i.e., assuming a constant relative velocity between the receivers over the correlation window). This motivates using noise bands with longer wavelengths to make the NCF more robust to receiver movement. Longer wavelengths are also frequently used in noise correlations because they have the most energy and travel longer distances. ${ }^{1}$ In our experiments the frequency band, $B_{\omega}=[100,500] \mathrm{Hz}$, was chosen by looking at the frequency content at the receivers and choosing bands with the largest energy. In this band, we expect shipping traffic and noise from shore to be the dominating noise sources. Typically the choice of bandwidth is limited by the environmental conditions of the receivers, we do not want to correlate over bands which do not pass through both receivers.

Given a set bandwidth, the choice of $T_{r}$ depends on the expected relative motion of the receivers. For our given 
wavelengths $(\lambda \approx[3,15])$ and rough estimates for relative motion (one to tens of centimeters per second) we are restricted to small values of $T_{r}$ (on the order of seconds) before we see peak degradation of the NCF. In the results of Fig. 2 and future figures we choose $T_{r}=10 \mathrm{~s}$, a conservative time window allowing for fairly large receiver motion (on the order of tens of centimeters per second).

Determining the optimal choice of $B_{\omega}$ and $T_{r}$ is nontrivial; there are two competing effects resulting from the choice of these parameters. On one hand, the choice of $B_{\omega}$ and $T_{r}$ defines a noise floor through the $\left(2 B_{\omega} T_{r}\right)^{-1}$ relationship that must be overcome to see a peak in the NCF. On the other hand, choosing large $B_{\omega}$ and $T_{r}$ will violate the stationary assumption and can cause interference in the correlation process.

To demonstrate this trade off we present two Figures. In Fig. 4 we show the measured noise of the NCF compared to the theoretical model, $\left(2 B_{\omega} T_{r}\right)^{-1}$. Here the noise of the NCF is defined by computing the variance of the NCF for $0.15>|\tau|>1 \mathrm{~s}$ for all center times, $t_{c}$, during the deployment and across all pairs. The time lags, $0.15>|\tau|>1$, were chosen because they are outside of all expected correlation peaks for all pairs. In Fig. 4 we show the average of the measured variance along with the standard deviation of this measurement. We provide the values for the theoretical model, $\left(2 B_{\omega} T_{r}\right)^{-1}$, for reference. We can see that the slope of the measured noise of the NCF is similar to the slope provided by the model, confirming that noise of the NCF decreases by a power law as $T_{r}$ is increased.

Figure 5 is provided to show how the peak of the NCF evolves as $T_{r}$ is adjusted. From our earlier analysis, we expect the peak in the NCF to increase as we increase $T_{r}$ until the distance between the receivers changes on the order

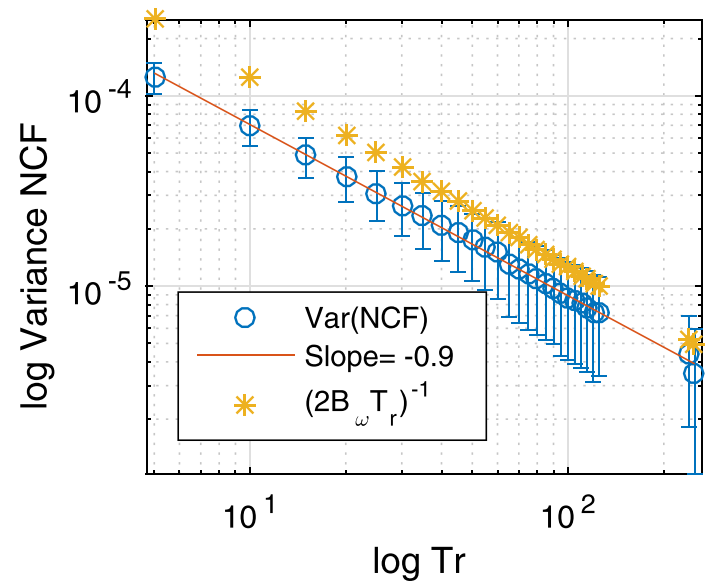

FIG. 4. (Color online) Variance of the NCF noise as a function of $T_{r}$. The variance of the NCF is computed between time lags 0.15 and $1 \mathrm{~s}$ in both positive and negative time for all pairs and all center times, $t_{c}$, in the deployment. This is done for different values of $T_{r}$, the correlation window length. The mean of all of the computed variance measurements is shown along with one standard deviation of these measurements. A best fit line is drawn for the mean of the measured values. The slope of the best fit line is -0.9 , which is close to the theoretical value of -1 , shown by (orange) asterisks, presented in Ref. 4. This plot shows that the noise in the NCF decays close to the predicted model as we vary the window length. This information is important in determining the optimal parameter for $T_{r}$ that depends on receiver movement and the noise floor.

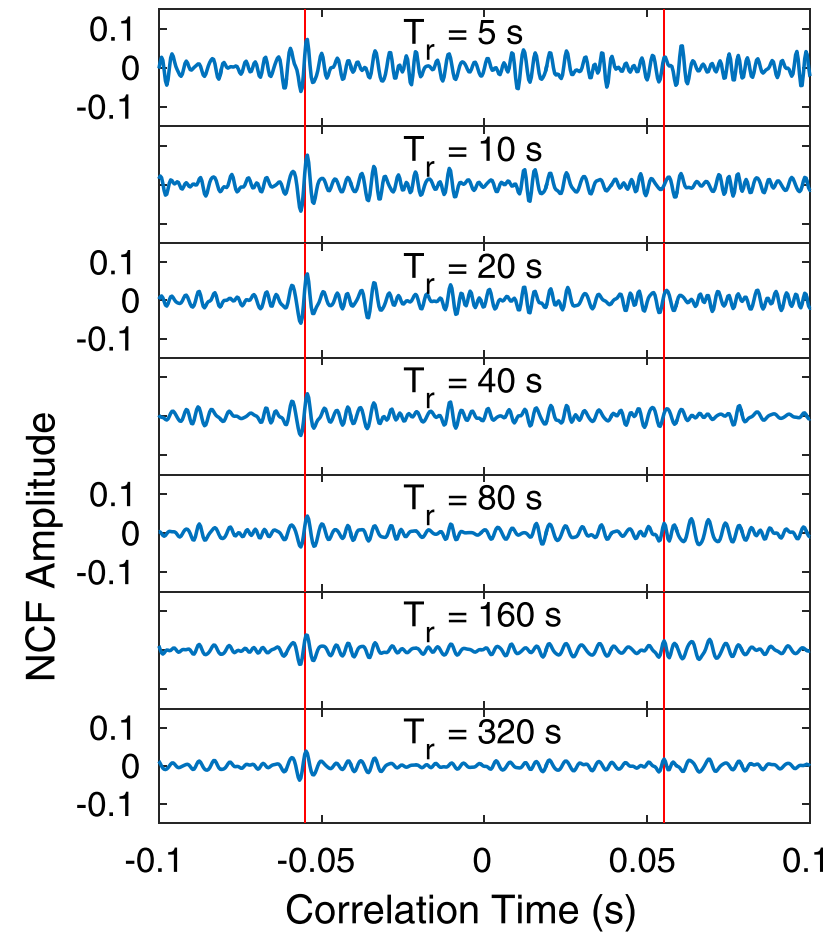

FIG. 5. (Color online) NCF peaks as a function of time window length $\left(T_{r}\right)$. The NCF is plotted for different lengths of correlations starting with $5 \mathrm{~s}$ and ending at $320 \mathrm{~s}$. All NCFs share a common center time of $t_{c}=1 \mathrm{~h} 30 \mathrm{~s}$. A red vertical line is placed where we expect the correlation peak to be based on receiver separation. We can see that as we increase the time window length, the variance of the NCF (i.e., the amplitude of the "noise") decreases. However, the peak on the NCF does not behave as nicely. We can see the peak in negative correlation time become more pronounced as we increase the window length from 5 to $20 \mathrm{~s}$ after which the amplitude of the peak starts to decline and become closer to the noise floor.

of a half wavelength. In this example, we can see that the $\mathrm{NCF}$ is one sided, meaning that there is only a peak in negative correlation time. As expected, the noise of the NCF decreases as $T_{r}$ is increased from 5 to $320 \mathrm{~s}$, agreeing with Fig. 4. Also, we can see the peak of the NCF in negative correlation time increase from $5 \mathrm{~s}$ until around $20 \mathrm{~s}$ before the peak begins to lose prominence. While $20 \mathrm{~s}$ may be close to an optimal choice in this example, it is difficult to determine this globally because it depends on the relative motion between the receivers as well as the noise field at that specific time. This motion is not constant and the noise field is not stationary. This is for one specific example in the deployment and while the intuition should translate to other cases, the optimal value for $T_{r}$ is expected to vary based on the noise distribution and relative movement.

Whether or not a peak will emerge in the NCF depends on the source distribution and noise coherence. Specifically, it will depend on whether or not there are noise sources whose components pass through both receivers and how much coherence is measured between the two receivers based on this propagation. We have already seen that this is not a constant process. There are times during the deployment where there is stronger coherence between the receivers and there are also directions where the noise propagation between the receivers results in stronger correlation peaks. In summary, the choice of $B_{\omega}$ and $T_{r}$ depends on the 
source distribution, the source level and the relative movement between the receivers. All of these environmental conditions are time varying, and often unknown.

\section{PROPAGATION ENVIRONMENT}

The acoustic environment of the AUEs during the deployment was not ideal for the propagation of the ambient sound field between the elements of the AUE array. The AUE's depth target, $10 \mathrm{~m}$, was on the boundary of the mixed layer and a steep thermocline, providing a strong downward refracting profile (Fig. 6). The shallow depth target allowed surface reflections of the noise field to interfere with the direct path (i.e., the environment is a Lloyd's mirror). Figure 6 describes the propagation environment of the receivers based on time averaged temperature data collected every $15 \mathrm{~min}$ near the array. In panel (a) the sound speed profile is given. In panel (b) we see the transmission loss calculated for the center frequency $(300 \mathrm{~Hz})$ using the parabolic equation method. ${ }^{24}$ This panel shows transmission loss colored in decibels, demonstrating the difficulty of horizontal transmission at $10 \mathrm{~m}$ depth. Additionally, the eigenrays, which are valid propagation paths between receivers given the sound speed profile, are plotted for different values of receiver separation in the horizontal direction. The eigenrays show the surface reflected path as well as the downward diffracting ray paths. Both of these demonstrate the difficulty of ambient noise propagating between the receivers during this deployment and suggest that more favorable environmental conditions are possible.

Panel (c) of Fig. 6 shows the transmission loss of the measured amplitude of the NCF from samples of correlation data (e.g., data from pairs like those shown in Fig. 2), as well as the frequency averaged (for the $B_{\omega}=[100,500] \mathrm{Hz}$ we are using) theoretical transmission loss generated from the parabolic equations. The measured amplitude was generated by taking the maximum value of the NCF for each short time cross correlation [i.e., the rows of Figs. 2(a) and 2(b)] indexed by binning the distance between the receivers at each time (the bin size used was $2 \mathrm{~m}$ ). All possible pairs of receivers were used in this analysis. Both the mean and the standard deviation (in log scale) are shown in this plot. The amplitude of the correlation function has large variance, highlighting the inconsistencies of the noise field during the deployment and the deviations from the isotropic noise field that theoretically recovers the amplitude of the TDGF. Even though the variance of the amplitude is large, averaging over many samples of the amplitude retrieves a power law. This result is encouraging. On average, we are recovering amplitude information between the different receivers, that is, information of the transmission loss between receivers. While the complicated environment makes it difficult to say, anything about the environment with certainty, there does seem to be an underlying physical process between the receivers.

We would expect the NCF to recover the amplitude of the TDGF only under an isotropic distribution of noise sources. ${ }^{3}$ The theoretical transmission loss indicates greater attenuation than what is observed in the data. The smaller attenuation in the observed data indicates that the coherent contributions are coming from the far field, which is consistent with shipping and environmental noise sources that are expected in the lower frequency bands. Additionally, the frequency whitening and amplitude clipping that was performed in the computation of the NCF to spread the coherent arrivals over multiple sources, with the intention of making the noise field more "isotropic," may also alter the amplitude of the NCF. What is interesting is that we do see a power law for amplitude decay as a function of increased distance. We believe that this indicates that there is some physical information embedded in the amplitude of the NCF.

\section{INCOHERENT BEAMFORMER}

In Sec. III we concluded that we could not recover the arrival structure of the TDGF from the NCF between some receiver pairs because of an imbalance of the noise distribution in the environment. In this section, we assume that there is a dominant source direction and we provide a technique to detect its presence as well as its direction. Coherent beamforming is a typical solution to this problem, yet coherent
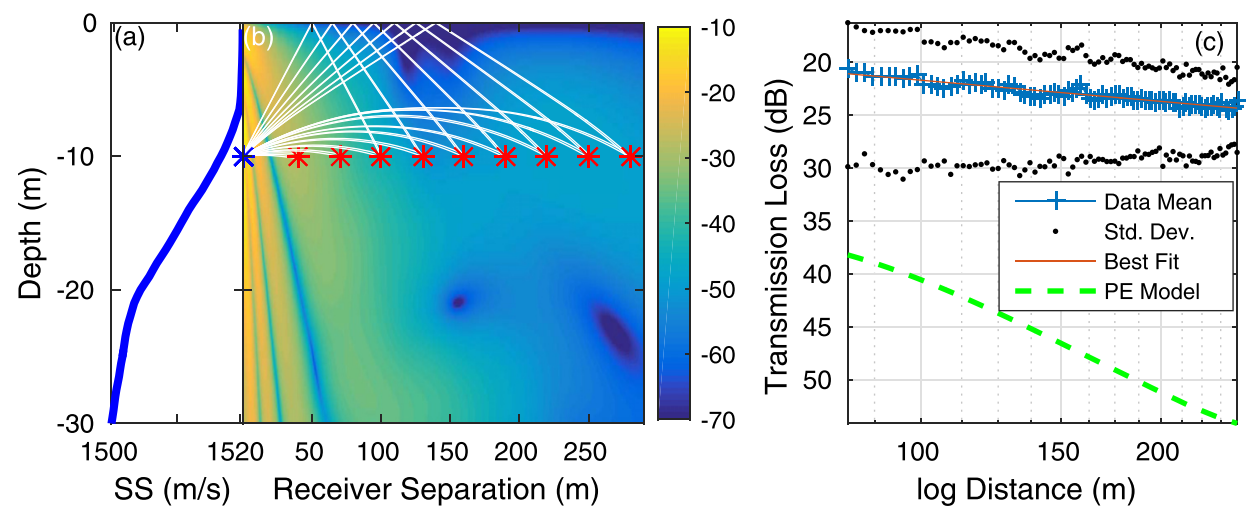

FIG. 6. (Color online) Environmental conditions: (a) sound speed profile averaged over 15 min intervals during the deployment. (b) Eigenrays for a sample of horizontal distances between receivers, we can see a surface reflection appear at distances close to $100 \mathrm{~m}$ that may cause interference with the direct path. Beneath the eigenrays, the transmission loss is shown (in $\mathrm{dB}$ ) as the solution to the parabolic equation. Both the eigenray and the transmission loss are computed for a frequency of $300 \mathrm{~Hz}$. (c) Transmission loss for both the measured data (along with the standard deviation for each distance bin) as well as the theoretical values from the parabolic equation. This plot demonstrates the challenges of horizontal propagation in the experiment's environment as well as the fact that on average, we recover a power law from the observed data. 
beamforming would not work well here because (1) the spacing between receivers is on the order of tens to hundreds of wavelengths whereas coherent beamforming typically requires spacing on the order of $1 / 2$ wavelength to avoid aliasing issues, (2) the position of the receivers are only estimated and are not precise enough for any type of coherent processing, and (3) the configuration of the array is ad hoc and does not follow any optimal pattern. To overcome these limitations, we introduce an incoherent beamformer. We show that we are able to recover the dominant noise directions received by the ad hoc array of receivers using the noisy localization results from the high frequency pinging system, and we show how these detected directions are consistent with our analysis in Sec. III.

To describe the incoherent beamformer, we start with the observation that given a plane wave representation of the dominant source, a peak will arise at time $\delta_{p}$ in the NCF between receiver $i$ and receiver $j$ according to

$$
\delta_{p}=\frac{P_{\theta}\left(S_{j}-S_{i}\right)}{c}+\nu
$$

where $P_{\theta}(\cdot)$ is the projection onto the direction of the dominant source (parameterized by $\theta$ ), $S_{i}$ and $S_{j}$ are the positions of receiver $i$ and $j, c$ is the speed of sound underwater, and $\nu$ is a noise term. The noise term, $\nu$, comes from inaccuracies in the estimated positions, $S_{i}$ and $S_{j}$, as well as violations of the plane wave assumption. Each NCF gives a measurement of the difference in distance of each receiver along the direction of propagation of the plane wave. We can build an incoherent beamformer by (1) assuming a dominant noise direction modeled by a plane wave, (2) estimating where the peaks in the NCF would be based on Eq. (2), (3) time shifting the envelope of the NCF so that the expected peak would be at 0 correlation time for all pairs, (4) average the NCFs from all pairs. Here we are working with the envelope of the NCF because of the challenges described with phase coherent processing. With this formulation, if there is a dominant source coming from the assumed direction and this dominant direction can be modeled by a plane wave then we would expect all of the peaks in the NCFs to average coherently at 0 correlation time. If the dominant source is not coming from the assumed direction, then time shifting the peaks will provide peaks that are not centered around zero and the peaks will not average coherently.

Using the estimated positions of each receiver $\left(S_{i}\right.$ and $S_{j}$ ) from the high frequency pinging system and assuming a dominant source direction, $\theta$ (in this case $45^{\circ}$, or northeast), Fig. 7(a) shows the time shifted envelopes using the procedure described. The peaks in this plot would line up close to zero time if the dominant direction was supported. We can see that this is not the case for the direction assumed in Fig. 7(a). In contrast, Fig. 7(b) shows the same plot for a different assumed direction (east) and we can see the peaks align around zero. This represents an agreement of the time-shifted correlations. To further quantify this agreement,
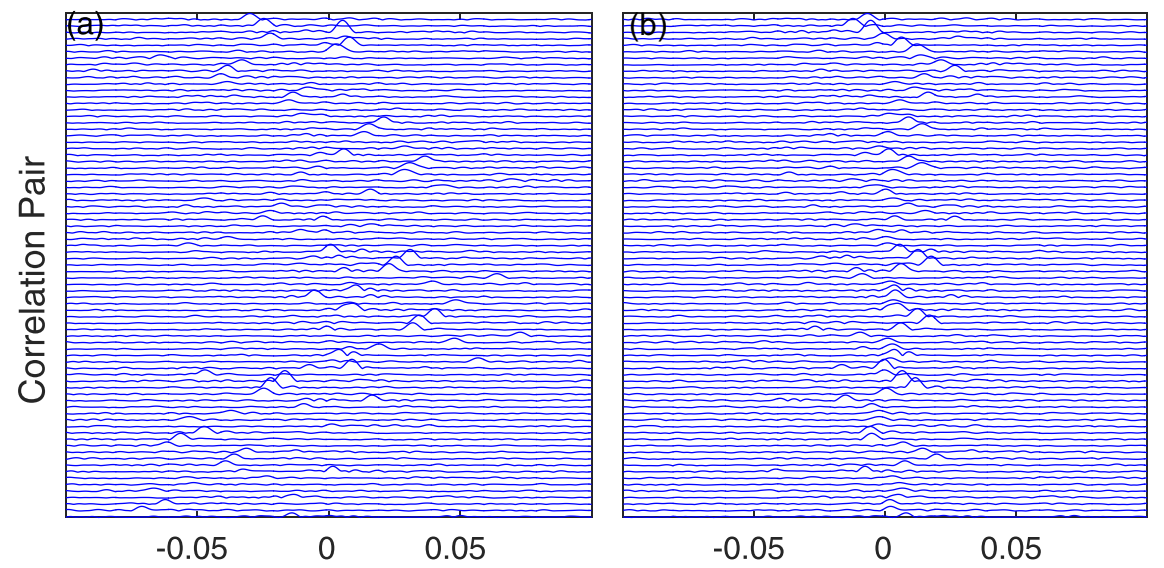

Time-Shifted Correlation Time (s) Time-Shifted Correlation Time (s)
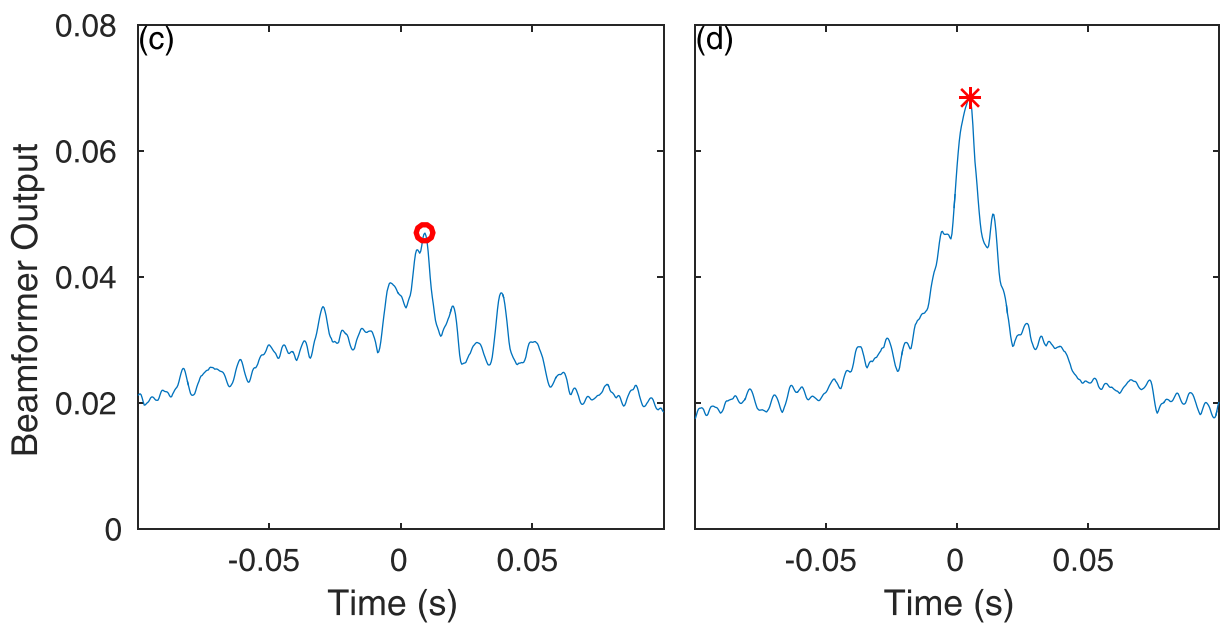

FIG. 7. (Color online) Illustrations of the incoherent beamformer procedure. A dominant noise direction is assumed [(a) $45^{\circ}$ or northeast, (b) east] and the NCF is time shifted so that the estimated location of the peak is at 0 correlation time. The locations of the receivers and an assumed noise direction are used to calculate this time shift. When the peaks align around 0 correlation time, such as in (b), their average at 0 correlation time is large. The average for (b) is shown in (d). When the peaks are not aligned (a), the average of the peaks at 0 correlation time is small. The average of (a) is shown in (c). We take the max of the average at within $10 \mathrm{~ms}$ of zero correlation time (to account for some error in the system) to be the output of the incoherent beamformer. When the output is large we suggest that the assumed dominant noise direction is supported (in this case directly east). When the dominant noise direction is small, the dominant noise direction is not supported. 
Figs. 7(c) and 7(d) show the average of the peaks in 7(a) and 7(b), respectively. In Figs. 7(c) and 7(d) the maximum is chosen to be the maximum value of the average within $10 \mathrm{~ms}$ of 0 correlation time. We believe $10 \mathrm{~ms}$ adequately accounts for the error in the system. We call this maximum the output of the incoherent beamformer.

We can perform this calculation for many assumed dominant noise directions to get an idea of the distribution of dominant noise sources for a given time during the deployment. Figure 8 shows this result for all directions during the same time that was used in the analysis of Fig. 7. Here we can see that the dominant noise direction is coming directly from the east because this gives us the largest output for the incoherent beamformer. Additionally, east looks like the only direction of dominant source during this time in the deployment because there is only one direction that produces a large output. Also highlighted are the angles that were shown in Fig. 7, east corresponds to Figs. 7(b) and $7(\mathrm{~d})$, while $45^{\circ}$ (northeast) corresponds to Figs. 7(a) and $7(\mathrm{c})$, respectively.

The output shown in Fig. 8 describes the environment of the array. In this case, the largest response from the incoherent beamformer is east, which is pointing directly towards shore. This is a typical response for this deployment and this supports our hypothesis that dominant directions in the noise field is the reason for symmetry being present in the NCF for some pairs, and not for others. Specifically, it supports the observation that the receivers with their endfire beam along the east-west direction would exhibit the arrival information of the TDGF because these noise directions pass through both receivers while the pairs whose endfire beams were oriented north-south do not exhibit this relationship. This is consistent with the analysis of Figs. 1 and 2 that were provided.

We can show the plot of Fig. 9 as a function of deployment time so that we can understand the time evolution of

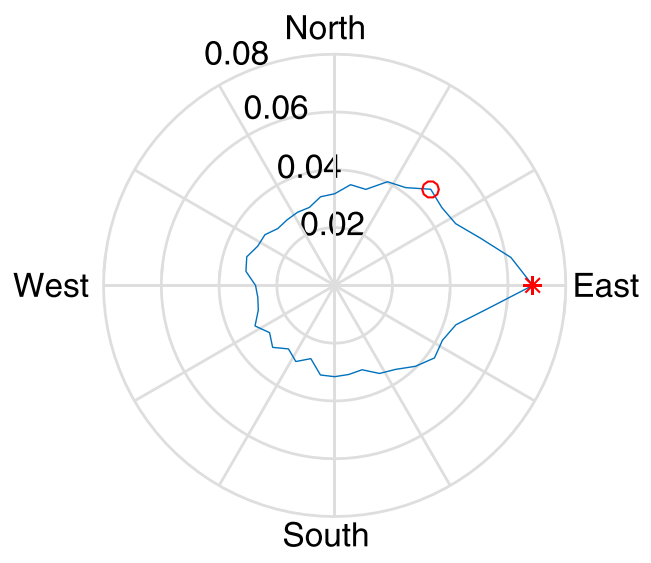

FIG. 8. (Color online) The incoherent beamformer output for one time during the deployment. We repeat the procedure of assuming a dominant noise direction and computing the output of the incoherent beamformer for all possible directions. This provides intuition of the dominant noise directions at a specific time. For example, at this particular time during the deployment, the majority of the noise field is coming from directly east of the array. This result supports our hypothesis that the noise field is not isotropic and is biased in the east-west direction. This is consistent with the observation in Fig. 2 that receiver pairs whose endfire beam are in the east-west directions demonstrate fair estimates of the arrival structure of the TDGF and receivers whose endfire is directed more in the north-south direction do not.

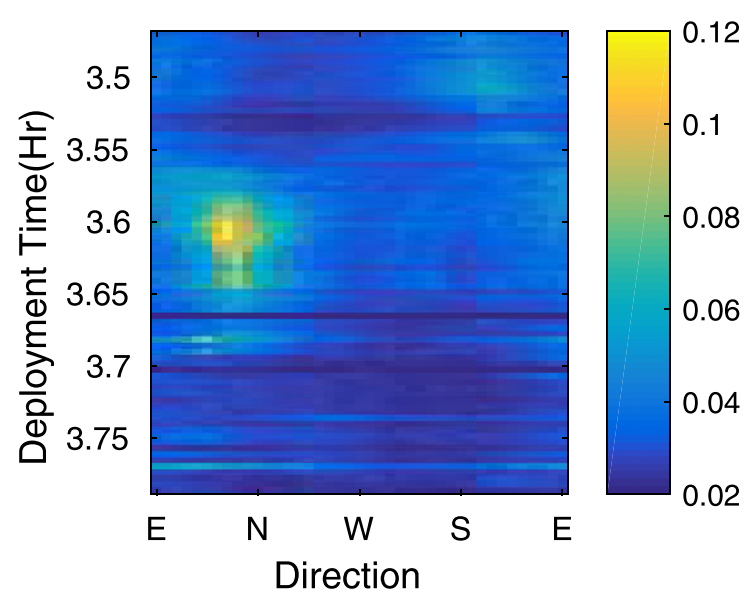

FIG. 9. (Color online) Time evolution of the beamformer output. Here each row is computed similar to Fig. 5 and is unrolled with the amplitude represented by the color shown on the color bar. We can see that there is a dominant direction of the ambient sound field just north of east. This is different than the dominant directions usually detected. We have verified that there is an identifiable boat in the recordings during this time so the presence of a directional bias in the ambient noise field is expected. This plot demonstrates the time varying nature of dominant noise directions during the deployment in $B_{\omega}=[100,500] \mathrm{Hz}$ band and this result is consistent with the arrival structure of the noise correlation function during this time in the deployment [shown by a green circle in Figs. 2(c) and 2(d)].

dominant signals in the ocean. Each row of Fig. 9 is the same information shown in Fig. 8 unrolled with the color showing the output amplitude of the incoherent beamformer. Like the NCF function presented in Fig. 2, these are also computed in $15 \mathrm{~s}$ intervals during the deployment. For this plot, we chose a period of time (approximately hour 3.5 to hour 3.8) where we have verified that there is an audible boat in the vicinity of the receivers. This boat provides dominant noise directions that are different from the rest of the deployment. We can see from Fig. 9 the direction of the incident noise coming from the boat and we can see that this lasts for less than $10 \mathrm{~min}$.

For the boat case, it is interesting to see how our assumption of a plane wave representation for the ambient noise holds up. We can see that the peak in this figure is spread over many values of $\theta$ which may indicate that we are not computing the appropriate time shifts from Eq. (2) because the source is too close to the array. This may hint that there may be able to be some improved detection by refining the estimate to include nearby sources (that cannot be modeled by a plane wave). Nonetheless, we see that the incoherent beamformer is able to detect the presence of dominant noise signals even though the spacing between the receivers is large, the geometry of the array is not optimal for coherent processing and the positions of the receivers are not precisely known.

Figure 9 shows a dominant noise direction from the north as opposed to the typical easterly direction. If we revisit the NCF in Fig. 2 we can see the effect of this boat, highlighted by the green circles in the intensity plot. We can see that for a brief period of time, the arrival information matches with the pair whose endfire beam is in the northsouth direction and not for the pair whose endfire is in the east-west direction. This is also consistent with the arrival 
analysis of Fig. 3 where we can see that the arrival information is more accurate for the north direction during hour 3.5 to hour 3.9 (Fig. 3) than during other times in the deployment [Figs. 3(b) and 3(c)]. This again shows consistency of the incoherent beamformer observations with the results we obtain in the NCF.

\section{CONCLUSION}

We presented a new application of ambient acoustic noise processing using mobile receivers whose geometry deforms over time. In this challenging environment, we were able to extract information related to the travel time between pairs of receivers using only $10 \mathrm{~s}$ cross-correlations. The amplitude of the TDGF is generally not retrieved in ambient noise processing because of complications in the noise distribution. The average amplitude of the NCF follows a power law when tracked over varying distances of receiver pairs demonstrating that the amplitude may follow some physical model. We developed an incoherent beamformer that we used for source localization. We also used the incoherent beamformer to detect the direction of anisotropy, which was consistent with our analysis of the noise correlation function.

\section{ACKNOWLEDGMENTS}

This work is part of "INSPIRE Track I: Distributed Sensing Collective to Capture 3D Soundscapes" supported by the NSF under Grant No. 1344291. This material is also based upon work supported by NSF Grant No. OCE 0927449, the National Science Foundation Graduate Research Fellowship under Grant No. DGE-1144086, the NSF Graduate Research Opportunities Worldwide program, the Chateaubriand STEM Fellowship, and the Friends of the International Center Scholarship at UCSD. ISTerre is part of LabEx OSUG@2020.

${ }^{1}$ Philippe Roux, W. A. Kuperman, and Karim G. Sabra, "Ocean acoustic noise and passive coherent array processing," C. R. Geosci. 343(8), 533-547 (2011).

${ }^{2}$ Philippe Roux, W. A. Kuperman, and the NPAL Group, "Extracting coherent wave fronts from acoustic ambient noise in the ocean," J. Acoust. Soc. Am. 116(4), 1995-2003 (2004).

${ }^{3}$ Philippe Roux, Karim G. Sabra, W. A. Kuperman, and Andre Roux, "Ambient noise cross correlation in free space: Theoretical approach," J. Acoust. Soc. Am. 117(1), 79-84 (2005).

${ }^{4}$ Karim G. Sabra, Philippe Roux, and W. A. Kuperman, "Emergence rate of the time-domain greens function from the ambient noise cross-correlation function," J. Acoust. Soc. Am. 118(6), 3524-3531 (2005).

${ }^{5}$ Karim G. Sabra, Philippe Roux, Aaron M. Thode, G. L. DSpain, W. S. Hodgkiss, and W. A. Kuperman, "Using ocean ambient noise for array self-localization and self-synchronization," IEEE J. Ocean. Eng. 30(2), 338-347 (2005).
${ }^{6}$ Oleg A. Godin, "Emergence of deterministic greens functions from noise generated by finite random sources," Phys. Rev. E 80(6), 066605 (2009).

${ }^{7}$ Karim G. Sabra, Philippe Roux, and W. A. Kuperman, "Arrival-time structure of the time-averaged ambient noise cross-correlation function in an oceanic waveguide," J. Acoust. Soc. Am. 117(1), 164-174 (2005).

${ }^{8}$ Karim G. Sabra, Bruce Cornuelle, and W. A. Kuperman, "Sensing deepocean temperatures," Phys. Today 69(2), 32-38 (2016).

${ }^{9}$ Katherine F. Woolfe, Shane Lani, Karim G. Sabra, and W. A. Kuperman, "Monitoring deep-ocean temperatures using acoustic ambient noise," Geophys. Res. Lett. 42(8), 2878-2884, doi:10.1002/2015GL063438 (2015).

${ }^{10}$ Peter Gerstoft, William S. Hodgkiss, Martin Siderius, Chen-Fen Huang, and Chris H. Harrison, "Passive fathometer processings," J. Acoust. Soc. Am. 123(3), 1297-1305 (2008).

${ }^{11}$ Martin Siderius, Heechun Song, Peter Gerstoft, William S. Hodgkiss, Paul Hursky, and Chris Harrison, "Adaptive passive fathometer processing," J. Acoust. Soc. Am. 127(4), 2193-2200 (2010).

${ }^{12}$ Martin Siderius, Chris H. Harrison, and Michael B. Porter, "A passive fathometer technique for imaging seabed layering using ambient noise," J. Acoust. Soc. Am. 120(3), 1315-1323 (2006).

${ }^{13}$ Laura A. Brooks and Peter Gerstoft, "Greens function approximation from cross-correlations of $20-100 \mathrm{~Hz}$ noise during a tropical storm," J. Acoust. Soc. Am. 125(2), 723-734 (2009).

${ }^{14}$ Shane W. Lani, Karim G. Sabra, William S. Hodgkiss, W. A. Kuperman, and Philippe Roux, "Coherent processing of shipping noise for ocean monitoring,” J. Acoust. Soc. Am. 133(2), EL108-EL113 (2013).

${ }^{15}$ Charlotte Leroy, Shane Lani, Karim G. Sabra, William S. Hodgkiss, W. A. Kuperman, and Philippe Roux, "Enhancing the emergence rate of coherent wavefronts from ocean ambient noise correlations using spatiotemporal filters," J. Acoust. Soc. Am. 132(2), 883-893 (2012).

${ }^{16}$ Stephanie. E. Fried, W. A. Kuperman, Karim G. Sabra, and Philippe Roux, "Extracting the local greens function on a horizontal array from ambient ocean noise,” J. Acoust. Soc. Am. 124(4), EL183-EL188 (2008).

${ }^{17}$ Karim G. Sabra, Stephanie Fried, W. A. Kuperman, and Mark Prior, "On the coherent components of low-frequency ambient noise in the Indian Ocean,” J. Acoust. Soc. Am. 133(1), EL20-EL25 (2013).

${ }^{18}$ Christoph Sens-Schonfelder, "Synchronizing seismic networks with ambient noise," Geophys. J. Int. 174(3), 966-970 (2008).

${ }^{19}$ Diba Mirza, Perry Naughton, Curt Schurgers, and Ryan Kastner, "Realtime collaborative tracking for underwater networked systems," Ad Hoc Netw. 34, 196-210 (2014).

${ }^{20}$ Jinwang Yi, Diba Mirza, Ryan Kastner, Curt Schurgers, Paul Roberts, and Jules Jaffe, "Toa-ts: Time of arrival based joint time synchronization and tracking for mobile underwater systems," Ad Hoc Netw. 34, 211-223 (2015).

${ }^{21}$ Robert Pinkel, M. A. Goldin, J. A. Smith, O. M. Sun, A. A. Aja, M. N. Bui, and T. Hughen, "The wirewalker: A vertically profiling instrument carrier powered by ocean waves," J. Atmos. Ocean. Technol. 28(3), 426-435 (2011).

${ }^{22}$ Laurent Stehly, Michel Campillo, Brnice Froment, and Richard L. Weaver, "Reconstructing Greens function by correlation of the coda of the correlation (c3) of ambient seismic noise," J. Geophys. Res.: Solid Earth 113, B11306, doi:10.1029/2008JB005693 (2008).

${ }^{23}$ Bernice Froment, Michel Campillo, and Philippe Roux, "Reconstructing the Greens function through iteration of correlations," C. R. Geosci. 343(89), 623-632 (2011).

${ }^{24}$ Finn B. Jensen, William A. Kuperman, Michael B. Porter, and Henrik Schmidt, in Computational Ocean Acoustics (Springer, New York, 2011), Chap. 6.

${ }^{25}$ J. S. Jaffe, P. J. S. Franks, P. L. D. Roberts, D. Mirza, C. Schurgers, R. Kastner, and A. Boch, "A swarm of autnomous miniature underwater robot drifters for exploring submesoscale ocean dynamics," Nat. Commun., in press (2017). 